\title{
Inventaire 1986 des Chironomidés (Diptera) connus des Pyrénées
}

H. Laville!
G. Vinçon'

Mots clés : Diptera, Chironomidae, faunistique, écologie, massif montagneux.

Un nouvel inventaire des Chironomidés recensés dans les pyrénées est présenté, à la lumière de récoltes récentes effectuées dans plusieurs vallées des versants français et espagnol. Cette étude a permis de porter à 288 le nombre d'espèces de Chironomidés actuellement connues des Pyrénées.

Sur les 56 especces $\left({ }^{\star}\right)$ nouvellement citées pour les Pyrénées, $12\left(^{\star \star}\right)$ sont également nouvelles pour la France.

1986 check-list of Chironomidae (Diptera) occurring in the Pyrenees.

heywords: Diptera, Chironomidac, faunistic, ecology, mountains.

A new list of chironomids recorded in the Pyrenees is presented, and utilises new information from several valleys on the French-Spanish border. The total number of chironomid species recorded in the Pyrenees is now 288 .

of the 56 species $\left({ }^{\star}\right)$ citcd for the first time for the Pyrenees, 12 species $\left({ }^{\star \star}\right)$ are also new records for France.

\section{Introduction}

La compilation de listes d'espèces d'une région géographique particulière constitue une étape fondamentale à toute recherche biogéographique et écologique.

Depuis le dernier inventaire (Laville 1980), la connaissance des Chironomidés pyrénéens a beaucoup progressé grâce d'une part aux travaux de plusieurs chercheurs français ou espagnols et d'autre part à l'évolution de la taxonomie du groupe.

Vu le nombre important des espèces nouvellement citées dans des travaux récents et souvent inédits de la chaine pyrénéenne, il nous a paru intéressant de présenter ce catalogue remis à jour, avant la parution de la nouvelle édition de la Limnofanma Europala.

1. Laboratoire d'Hydrobiologie, UA 695 C.N.R.S., Université Paul Sabatier, 118 Route de Narbonne, 31062 Toulouse Cédex, France.

\section{Travaux récents sur les Chironomi- dés pyrénéens.}

Les travaux parus après 1980 et analysés pour cet inventaire sont les suivants:

A) Versant espagnol

- Prat (1981).- Faune chironomidienne de quelques lacs et cours d'eau de Catalogne espagnole : 23 espèces sont recensées, 18 en basse altitude $(175 \mathrm{~m}-310 \mathrm{~m}), 9$ en moyenne ou haute montagne (>1000 m).

- Gonzalez-Peña (1983).- Chironomidés du réseau hydrographique du Rio Sègre (Andorre), entre $800 \mathrm{~m}$ et $2000 \mathrm{~m}: 43$ taxons sont recensés dont 20 identifiés à l'espèce et 6 au groupe d'espèces.

- Prat \& al. (1984).- Répartition altitudinale des Chironomidés du Rio Llobregat (Catalogne espagnole). de $1500 \mathrm{~m}$ jusqu'au niveau de la mer: liste faunist $\mathrm{j}$ que de 15 espèces et $\mathbf{4}$ taxons identifiés au groupe d'espèces. 
- Rieradevall i Sant (1985).- Etude intensive du peuplement chironomidien du Rio Llubregat à $320 \mathrm{~m}$ : 49 espèces signalées dans une seule station.

\section{B) Versant français}

- Thomas (1981).- Faune macroinvertébrée du fleuve Garonne, en amont et en aval de l'usine de cellulose de Saint-Gaudens $(340 \mathrm{~m})$ : liste faunistique de 46 espéces de Chironomidés (Détermination J.-N. Tourenq).

- Gazagnes \& Laville (1985).- Faune chironomidienne de la Haute Neste d'Aure entre $1080 \mathrm{~m}$ et $1440 \mathrm{~m}$ : liste faunistique de 65 taxons dont 51 identifiés à l'espèce.

- Brouquet- Laglaire (1985).- Les Chironomidés du Touyre, sous-affluent de la rivière Ariège, entre 330 $m$ et $670 \mathrm{~m}: 72$ espèces recensées (Détermination H. Laville).

\section{C) Travaux inédits}

A ces données récentes nous avons joint des résultats encore inédits de récoltes provenant de trois vallées pyrénéennes du centre ei de l'ouest de la chaine.

- Basse Neste d'Aure dans les Pyrénées centrales, entre $420 \mathrm{~m}$ et $1060 \mathrm{~m}$ (Bouguenec \& al. 1984 : dét. H. Laville) : sur les 39 espèces recensées dans ce travail non publié, 20 n'étaient pas signalées de la Haute Neste d'Aure (Gazagnes \& Laville 1985). Leur liste est récapitulée sur le tableau I. Toutes sont regroupées dans le catalogue général (Tableau II) dans la même colonne (H) attribuée à la Neste d'Aure dans son ensemble.

- Vallée d'Ossau dans les Pyrénées atlantiques (Vinçon 1987) : 111 espèces recensées entre $450 \mathrm{~m}$ et 2150 m (Dét. H. Laville \& B. Serra-Tosio ${ }^{1}$ : Diamesinae).

- Haute vallée du Rio Gallego, affluent de l'Ebre, à $1750 \mathrm{~m}$ (Vinçon 1987 : dét. H. Laville) : 16 espèces rencontrées dans deux stations.

\section{Donnêes faunistiques}

L'inventaire faunistique (Tableau II) est présenté sous forme d'un catalogue avec la nomenclature de la Limnofama Europaea (Fittkau \& Reiss 1978) actualisée conformément aux travaux taxonomiques plus récents (Tableau III)

1. Nous remercions B. Serra-Tosio (Grenoble) d'avoir bien voulu vérifier nos identifications des Diaunesinae des vallées d'Aure et d'Ossau.
Selon le code de la Limmofauna, les espèces rencontrées effectivement dans la zone $2(>1000 \mathrm{~m})$ des Pyrénées sont signalées par un gros point noir $(\bullet)$ : celles présentes seulement en bordure de la chaine $(<1000 \mathrm{~m})$ sont marquées d'un point plus petit (.).

Les espèces dont la répartition diffère de l'édition 1978 de la Limmotatma sont sigalées par une flèche $\rightarrow$. Les différences les plus courantes concernent les nouvelles citations et les espèces qui, jusqu'ici, n'ont été rencontrées qu'en bordure de la chaine $(300 \mathrm{~m}$ - $1000 \mathrm{~m}$ ) et qui, de ce fait, n'appartiennent pas à la zone $2\left(Z_{2}\right)$ sensit stricto.

Cinq espèces ont été retirées de ce catalogue, leur citation antérieure dans la zone 2 pyrénéenne paraissant erronée ou douteuse.

- Cryplocladopelma laccophila $\mathbf{K}$. et Dicrotendipes tritomus K. sont seulement signalées de Gallice (Espagne) du Lago de Lacillos (Zamora) par Bertrand (1956); elles ont été citées par erreur des Pyrénées (Laville 1980).

- Micropsecira non bodanica Reiss: Micropsecra jumci (Mg.) (Laville \& Lavandier 1977).

- Natarsia nugax (Walk.) et Paratanyarsus natrigi (G.) : leur localisation dans les Pyrénées n'a pas été retrouvée. $N$. nugax est vraisemblablement signalée pour N. punctata (Fabr.).

Quelques espèces, identifiées de façon incertaine, ne sont pas incluses dans cet inventaire :

Gazagnes \& Laville (1985) :

- Cricotopus of albiforceps (K.)

- Connoneura cf edwardsi $\mathrm{Br}$.

Vinçon (1987):

- Metriocnentis of terrester Pag.

- Chironomus of bernensis Wülk. \& Klöt.

\section{Résultats}

12 espèces précédées de deux astérisques $\left({ }^{\star \star}\right)$ sont nouvelles pour la Faune de France, 44 autres, avec un astérisque $\left(^{\star}\right)$ sont nouvelles pour les Pyrénées. ce qui porte à 288 le nombre total des Chironomidés recensés à ce jour dans ce massif montagneux. Ils se répartissent ainsi dans les principales sousfamilles ou tribus : 27 Tanypodinae, 24 Diamesinae, 3 Prodiamesinae, 140 Orthocladiinae, 43 Chironomini, 51 Tanytarsini (Tableau IV). 
Tableau I. Inventaire 1986 des Chironomidés des Pyrénées.

Citations récentes pour les Pyrénées: A : Laville (1980, inventaire), B : Prat (1981). C: Thomas (1981), D: Gonzalez-Pena (1983), E : (Prat \& al. 1984). F : Rieradevall i Sant (1985), G : Brouquet-Laglaire (1985), H : Bouguenec \& al. (1984) et Gazagnes \& Laville (1985), I : Vinçon (1987, Vallée d'Ossau), J : Vinçon (1987, Rio Gallegò à 1750 m), $\mathrm{K}:$ autres citations : (l) : Laville \& Lavandier (1977), (2) : Laville (1978), (3) : Laville (1966).

- Espèce présente dans la zone $2\left(Z_{2}\right)$ de la Limmotatma Etropaea (1978) (Pyrénées au-dessus de $1000 \mathrm{~m}$ ).

- espèce présente en bordure de la zone 2 (Pyrénées au-dessous de $1000 \mathrm{~m}$ ).

Les espèces dont la répartition a été modifiée par rapport à celle de la Limmofatua Europaea sont indiquées par une flèthe $(\rightarrow)$.

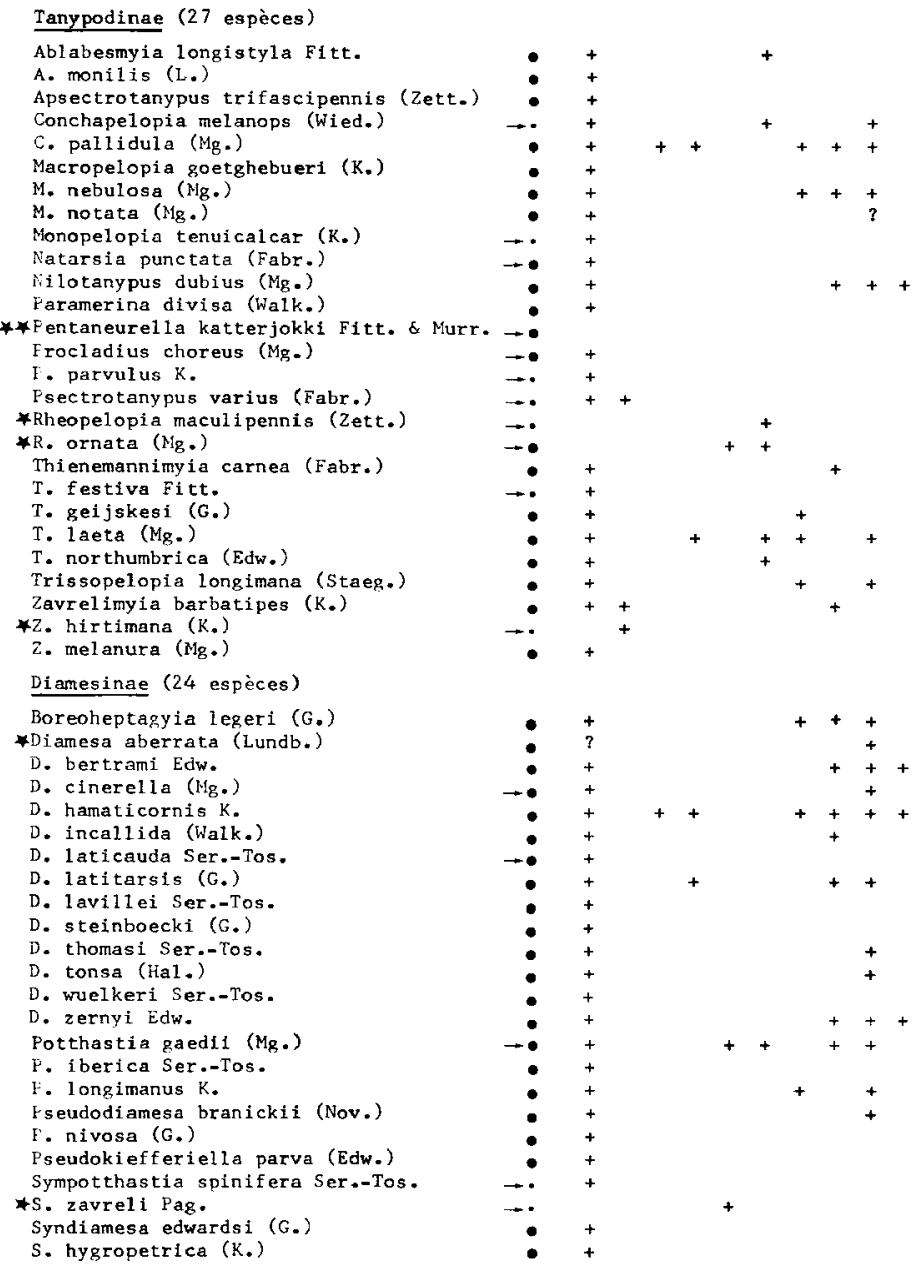


Prodiamesinae ( 3 espèces)

Monodiamesa ekmani $\mathrm{Br}$.

* Odontomesa fulva (K.)

Prodiamesa olivacea (Mg.)

Orthocladiinae (138 espèces)

Brillia longifurca $K$.

B. modesta ( $\mathrm{Mg.}$ )

Bryophaenocladius 111 imbatus (Edw.)

B. subvernalis ( $\mathrm{d} \mathrm{dw}$. )

Camptocladius stercorarius (de Geer)

* Cardiocladius capucinus (Zett.)

* C. fuscus (K.)

Chaetocladius dentiforceps (Edw.)

* *C. gracilis $\mathrm{Br}$.

C. Laminatus $\mathrm{Br}$.

*\#C. melal eucus ( $\mathrm{Mg}$.)

*C. perennis ( $\left.\mathrm{Mg}_{-}\right)$

* *C. suecicus (K.)

Corynoneura fittkaui Schlee

C. lacustris Edw.

C. Lobata Edw.

C. scutellata Winn.

Cricotopus annulator $G$.

C. bicinctus ( $\mathrm{Mg}$.)

*C. curtus Hirv.

*C. festivellus ( $\left.K_{*}\right)$

*C. flavocinctus (K.)

C. fuscus (K.)

*C. (Nostococladius) lygropis Edw.

*C. ornatus (Mg.)

*C. pilitarsis (Zett.)

c. pirifer Hirv.

\#C. similis $G$.

C. speciosus $\mathrm{G}$.

C. sylvestris (Fabr.)

C. tremulus (L.)

C. triannulatus (Macq.)

C. tricinctus ( $\mathrm{Mg}$. )

*C. trifascia Edy.

C. trifasciatus ( $\mathrm{Mg}$.)

*C. vierriensis $\mathrm{G}$.

* Diplocladius cultriger $\mathrm{K}$.

Epoicocladius flavens (Mall.)

Eukiefferiella brevicalcar (K.)

E. clari pennis (Lundb.)

E. clypeata (K.)

E. coerulescens $(K$.

E. cyanea Th.

E. devonica (Edw.)

E. fittkaui Lehm.

E. fuldensis Lehm.

E. gracei (Edw.)

*E. ilkleyensis (Edw.)

*E. lobifera G.

E. minor Edw.

*E. pseudomontana G.

$\begin{array}{llllllllllll}\mathrm{Z}_{2} & \text { A } & \text { B } & \text { C } & \text { D } & \text { E } & \text { F } & G & \text { H } & \text { I } & \text { J } & K\end{array}$

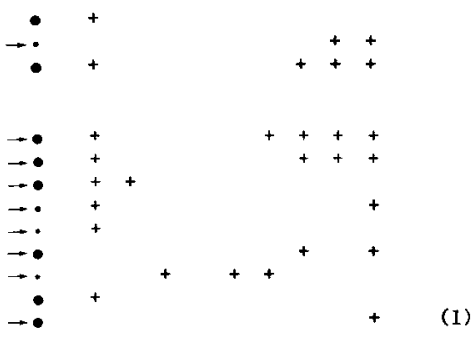

(2) 
Eukiefferiella similis $G$.

E. tirolensig $G$.

* Eurycnemus crassipes (Panz.)

Gyminometriocnemus brumalis (Edw.)

Heleniella ornaticollis (Edw.)

* Heterotanytarsus apicalis (K.)

Heterotrissocladius marcidus (Walk.)

Krenosmittia boreoalpina (G.)

\%K. camptophleps (Edw.)

Limophyes interruptus G.

L. gurgicola Edw.

L. minimus $(\mathrm{Mg} *)$

L. prolongatus (K.)

L. smolandicus $\mathrm{Br}$.

L. truncorum $G$.

Metriocnemus fuscipes ( $\mathrm{Mg}$ )

M. gracei Edw.

M. hirticollis (Staeg.)

$M$. hygropetricus $K$.

*M. picipes (Mg.)

\# M. tristellus Edw.

Nanocladius bicolor (Zett.)

N. parvulus (K.)

$N_{\text {. rectinervis (K.) }}$

Or thocladius s. 1 .

(Eudactylocladius) bi punctellus (Zett.)

(E.) fuscimanus $\mathrm{K}$.

(E.) tuberculatus (Edw.)

(Euorthocladius) rivicola (K.)

(E.) rivulorum (K.)

*(E.) saxosus (Tok.)

(E.) thienemanni (K.)

(Orthocladius) decoratus (Hoim.)

(0.) dentifer Br.

(0.) excavatus $\mathrm{Br}$.

(O.) frigidus (K.)

(0.) oblidens (Walk.)

(0.) rhyacobius (K.)

(0.) rubicundus $\left(\mathrm{Mg} g_{-}\right)$

(0.) saxicola (K.)

(0.) smolandicus Br.

* (Fogonocladius) consobrinus Holm.

Faracladius alpicola (Zett.)

P. conversus (Walk.)

Paracricotopus niger (K.)

P. uliginosus $\mathrm{Br}$.

Parakiefferiella bathophila (K.)

P. coronata (Edw.)

* F Farametriocnemus boreoalpinus Gow. P. stylatus (K.)

Paraphaenocladius penerasus (Edw.)

Faratrichocladius rufiventris ( $\mathrm{Mg}$. )

I. skirwithensis (Edw.)
$\begin{array}{llllllllllll}Z_{2} & \text { A } & \text { B } & \text { C } & \text { D } & \text { E } & \text { F } & \text { G } & \text { H } & \text { I } & J & \text { K }\end{array}$
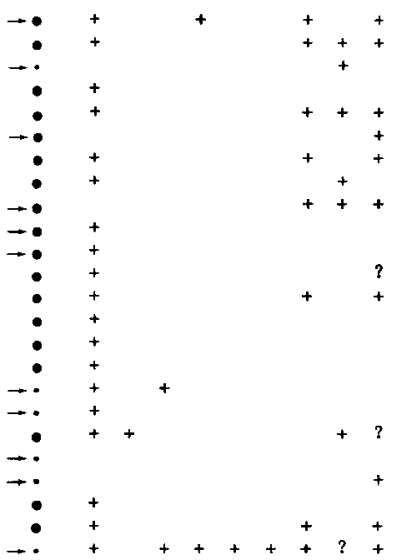
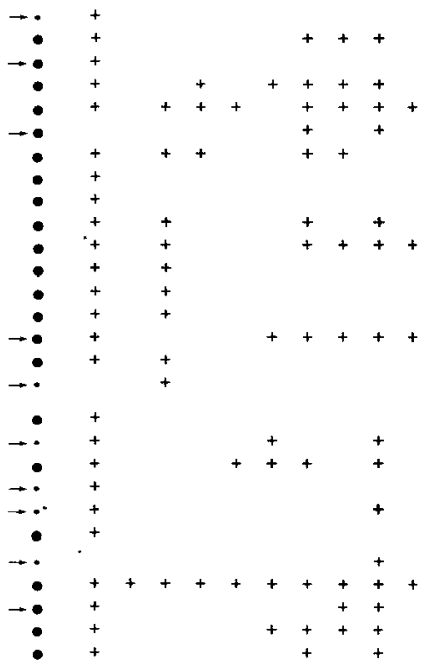
Paratrissocladius excerptus (Walk.)

Parorthocladius nudipennis (K.)

P. torrentium (G.)

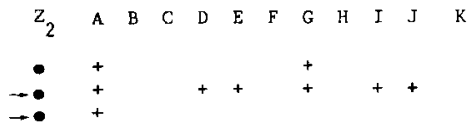

Psectrocladius s. 1.

(Allopsectrocladius) obvius (Walk (Mesopsectrocladius) barbatipes K. * (Monopsectrocladius) calcaratus Edw. (Psectrocladius) barbimanus Edw. *(F.) limbatellus (Holm.)

(F.) octomaculatus Wiilk.

(P.) oligosetus Wülk.

(P.) sordidellus (Zett.)

Pseudorthocladius curtistylus (G.)

Fseudosmittia gracilis (G.)

F. trilobata (Edw.)

Rheocricotopus atripes (K.)

* $R_{\text {. chalybeatus (Edw.) }}$

R. effusus (Wa1k.)

$R_{\text {. fuscipes (K.) }}$

* R. glabricollis ( $\mathrm{Mg}$.)

\# \#heosmittia spinicornis (Br.)

Smittia aterrima ( $\mathrm{Mg}$ )

S. Leucopogon (Mg.)

S. nudipennis $G$.

S. pratorum G.

Symbiocladius rhithrogenae K. \& Zav.

Synor thocladius semivirens (K.)

Thienemannia gracilis $K$.

* Thienemanniella acuticornis $\mathrm{k}$.

T. clavicornis $\mathrm{K}$.

T. flavescens Edw.

T. lutea Edw.

T. obscura Br.

*T. vittata Edw.

* Tveteria bavarica (G.)

T. calvescens (Edw.)

*T. discoloripes (G.)

T. veralli (Edw.)

$\bullet+$

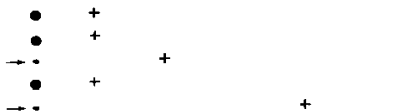

Chironominae ( 90 espèces)

CHIRONOMINI ( 43 espèces)

Chironomus anthracinus Zett.

C. cingulatus ( $\mathrm{Mg}$. )

C. commutatus Keyl.

C. plumosus L.

C. riparius $\mathrm{Mg}$.

Cladopelma lateral is G.

*Cryptochironoms rostratus

*C. supplicans ( $\mathrm{Mg}$. )

*Cryptotendipes nigronitens Edw.

C. pseudotener $G$.

Demicryptochironomus vulneratus (Zett.)

Dicrotendipes notatus ( $\mathrm{Mg}$ )

D. pulsus (Walk.) 
Einfelda dissidens (Walk.)

Endochironomus tendens Fabr.

Glyptotendipes gripekoveni K.

Microtendipes britteni Edw.

M. confinis ( $\mathrm{Mg}_{\text {. }}$ )

M. pedellus (de Geer)

M. rydalensis (Edw.)

Pagastiella orophila (Edw.)

paracladopelma camptolabis $K$.

F. laminata $\mathrm{K}$.

*H. mikiana G.

* F. nigritula G.

Faratendipes albimanus ( $\mathrm{Mg}_{\bullet}$ )

Phaenopsectra flavipes ( $\mathrm{Mg}$.)

Folypedilun s. 1 .

(Pentapedilum) nubens Edw.

(F.) sordens ( $\left.v . d_{*} W_{*}\right)$

(F) tritum (Walk.)

(Folypedilum) acutum $\mathrm{K}$.

(P.) a egyptium $K$.

(i ) albicorne (ig.)

* (F) apfelbecki (Strobl)

(F ) convictum (Walk.)

(F) cultellatum G.

(F) laetum ( $\mathrm{Mg}$.)

(I) nubeculosum (Yg.)

(F.) pedestre ( $\mathrm{kg}$ )

(F.) pullum (Zett .)

(P.) quadriguttatum $k$.

Stenochironomus gibbus Fabr.

Stictochironomus histrio (Fabr.)

TANYTARSINI (47 espèces)

Cladotany tarsus atridorsum (K.)

C. iucundus Hirv.

C. mancus (Walk.)

Krenopsectra fallax Reiss

Lithotanytarsus emarginatus $G$.

Nicropsectra apposita (Walk.)

M. atrofasciata K.

M. attenuata Reiss

Ni. bidentata G.

M. contracta Reiss

M. coracina (K.)

H. fusca $\mathrm{Mg}$.

M. junci ( $\mathrm{Mg}$. $_{\text {. }}$

M. 1 indrothi $G$.

M. notescens (Walk.)

M. recurvata $\mathrm{G}$.

Neozavrelia fuldensis Fitt.

Earatanytarsus austriacus $k$.

*F. bituberculatus (Edw.)

F. confusus Fal.

F. inopertus (Walk.)
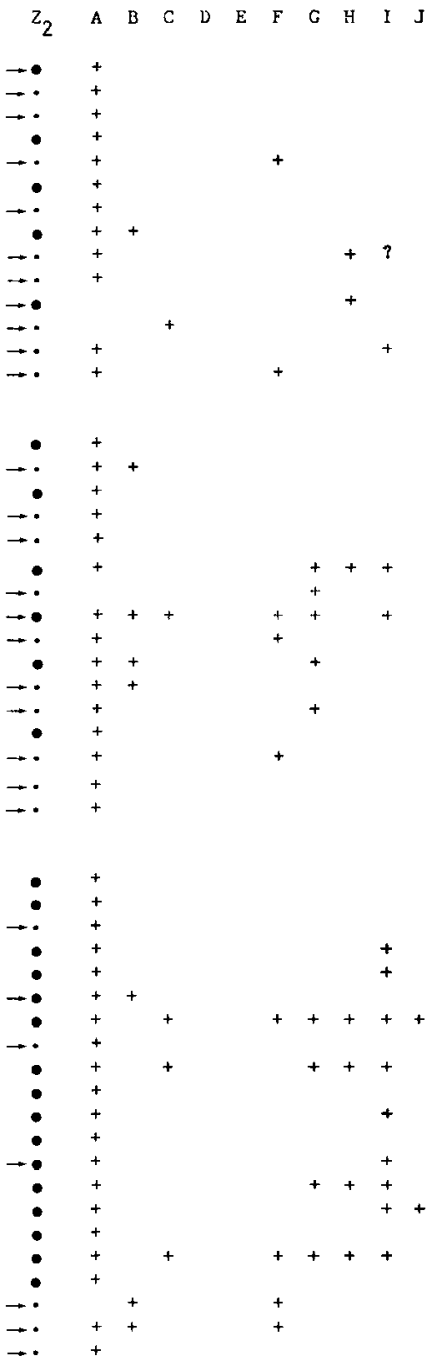

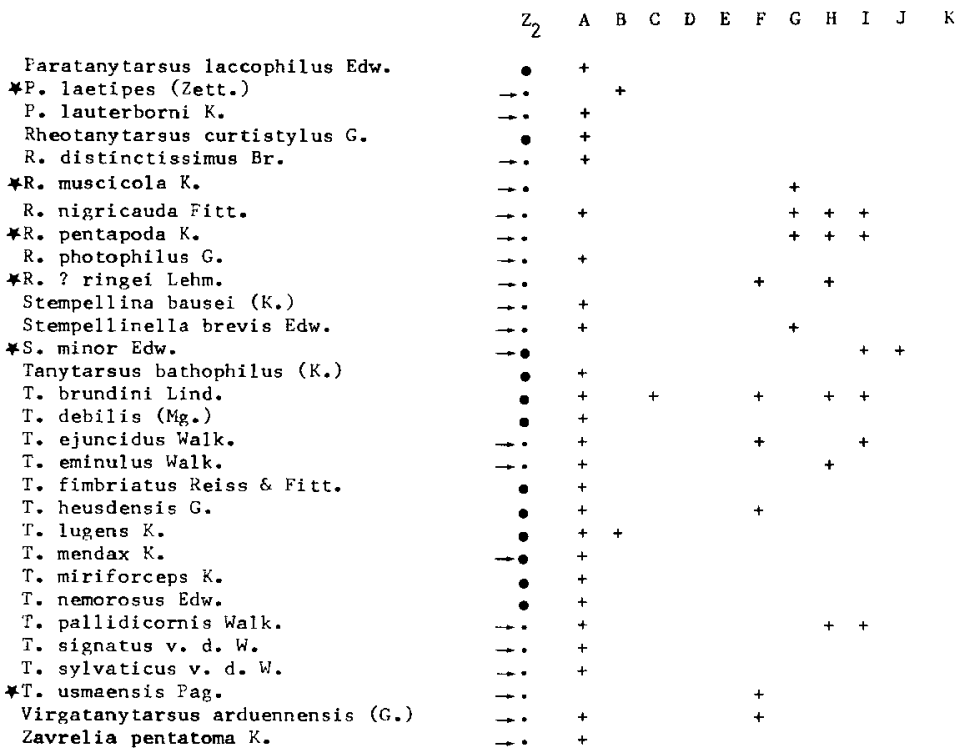

Tableau Il. Liste des 20 espèces récoltées dans la Neste d'Aure, seulement en basse altitude entre $400 \mathrm{~m}$ et $1000 \mathrm{~m}$, par Bouguenec \& al (1984)

Tanypodinae

Thienemannimyia carnea Zavrelimyia barbatipes

Diamesinae Pothhastia gaedii

Prodiamesinae Odontomesa fulva

Orthocladiinae Brillia longifurca Brillia modesta Cricotopus trifascia Eukiefferiella ilkleyensis Eukiefferiella tirolensis
Eurycnemus crassipes Paracricotopus niger Tvetenia veralli

Chironomini

Paracladopelma camptolabis

Tanytarsini

Micropsectra atrofasciata

Neozavrelia fuldensis

Rheotanytarsus pentapoda

Rheotanytarsus? ringei

Tanytarsus brundini

Tanytarsus eminulus

Tanytarsus pallidicornis 
Tableau III. Changements de nomenclature

\section{RÉFERENCES ANTERIEURES}

Laville \& Lavandier (1977)

Pentanetra sp Katterjokk Fitt.

Micopsectra non bodanica Reiss

I aville (1978)

Epoicocladius ephemerae $\mathbf{K}$.

Laville (1980)

Microcricotopus Thien, \& Harn.

Cryptocladopelma Lenz

Pentapedilum $\mathbf{K}$.

Bryophaenocladius flexidens $(\mathrm{Br}$.

Eukiefferiella calvescens Edw.

Eukiefferiella veralli Edw.

O. (Eudactylocladius) obtexens Br.

Rheocricotopus toveatus (Edw.)

Chironomus thummi $\mathrm{K}$.

Leptochironomus laminata $\mathbf{K}$.

Polypedilum scalaenum Schr.

Polypedilum vetterense $\mathrm{Br}$.

Lauterbornia coracina $\mathbf{K}$.

Tanytarsus arduennensis $\mathrm{G}$.

Tanytarsus holochlorus Edw.

Chaetocladius excerptus (Walk.)

Prat (1981)

Paratanytarsus handlirschi G.

Thomas (1981)

Eukiefferiella potthasti Lehm.

Eukiefferiella veralli (Edw)

Pogonocladius consobrinus (Holm.)

Rheocricotopus dispar $\mathrm{G}$.

Rheocricotopus foveatus (Edw.)

Rheocricotopus gouini $\mathrm{G}$.

Paracladopelma obscura $\mathrm{Br}$.

Brouquet-Laglaire (1985)

Trissocladius fluviatilis (G.)

Gazagnes \& Laville (1985)

Diamesa cf. vaillanti Ser. Tos.

En raison de la prospection plus intense des milieux lotiques, c'est surtout parmi les Orthocla. diinae que l'on rencontre le plus d'espèces nouvellement citées (36).

Pour connaître plus précisément la localisation des stations de récoltes de ces espèces, on pourra se reporter aux travaux des auteurs cités en référence dans le tableau $I$.

\section{Discussion d'espèces intéressantes}

Dans cette nouvelle liste quelques espèces particulières nécessitent des commentaires biogéographiques et écologiques.

\section{TRAVAIL PRESENT}

Pentaneurella kalterjokki Fitt. \& Mur. Micropsectra junci (Mg)

Epoicocladius flavens (Mall.)

Nanocladius $\mathrm{K}$.

Cladopelma K.

Polypedilum K. (S.g. Pentapedilum K.)

Bryophaenocladius illimbatus (Edw.)

Tvetenia calvescens (Edw.)

Tvetenia discoloripes (G.)

O. (Eudactylocladius) fuscimanus $\mathrm{K}$.

Rheocricotopus atripes (K.)

Chironomus riparius $\mathbf{M g}$.

Paracladopelma laminata $\mathbf{K}$.

Polypedilum quadriguttatum $\mathbf{K}$.

Polypedilum aegyptium $\mathrm{K}$.

Micropsectra coracina (K.)

Virgatanytarsus arduernensis (G.)

Tanytarsus mendax $\mathrm{K}$.

Paratrissocladius excerptus (Walk)

Paratanytarsus bituberculatus (Edw.)

Eukiefferiella gracei (Edw.)

Tvetenia discoloripes (G.)

Orthocladius (Pogonocladius) consobrinus (Holm).

Rheocricotopus fuscipes (K.)

Rheocricotopus atripes (K.)

Rheocricotopus glabricollis (Mg.)

Paracladopelma nigritula G.

Paratrissocladius excerptus (Walk.)

Diamesa zernyi Edw.

\section{ઐ $\star$ Pentaneurella katterjokki Fitt. \& Murray}

Cegenre monospécifique est seulement connu de Laponie suédoise et du Nord de la Norvège (Fittkau \& Murray 1983). Dans les Pyrénées, sa récolte dans la partie la plus froide (St. A : $2370 \mathrm{~m}$ ) du torrent d'Estaragne où la température est toujours inférieure à $4,5^{\circ} \mathrm{C}$, confirme sa sténothermie et révèle une répartition boréoalpine. Elle était signalée par erreur de la station D : $1850 \mathrm{~m}$ de l'Estaragne par Laville \& Lavandier (1977, sub. Pentaneura sp. Katterjokk Fitt.).

\section{Boreoheoptagyia legeri (G.)}

Syn. Heptagia punctulata G.

Boreoheptagia punctulata (G.) (Serra-Tosio 1980).

Espèce sténotope et sténotherme d'eau froide dont la larve vit dans les zones d'embruns des blocs de 
Tableau IV. Répartition des Chironomidés pyrénéens dans les principales sous-familles ou tribus, recensés en 1980 et 1986.

* Crptocladopelma laccophila K. et Dicrotendipes tritomus K., signalés par erreur dans l'inventaire 1980 ne sont plus comptabilisés.

\begin{tabular}{lccccccc} 
& Tanypodinae & Diamesinae & Prodiamesinae & Orthocladiinae Chironomini & Tanytarsini & Total \\
\hline $\begin{array}{l}\text { Inventaire 1980 } \\
\begin{array}{l}\text { Espèces nouvelles } \\
\text { pour les Pyrénées }\end{array}\end{array}$ & 23 & 22 & 2 & 104 & $37^{*}$ & 44 & $232^{*}$ \\
$\begin{array}{l}\text { s.l. } \\
\begin{array}{l}\text { Espèces nouvelles } \\
\text { pour la France }\end{array}\end{array}$ & 1 & 2 & - & 26 & 6 & 7 & 44 \\
Inventaire 1986 & 27 & 24 & 3 & 140 & 43 & 49 & 288 \\
\hline
\end{tabular}

pierre (Lehmann 1971). Bertrand (1952) la signale de petites cascades hygropétriques d'Andorre vers 1800-1900 m.

Répartition : Sud de l'Europe, Mittelgebirge, Alpes, Madere, Afrique du Nord (Lehmann loc. cit.), Pyrénées, Balkans orientaux, Caucase (Fittkau \& Reiss 1978).

En Espagne, elle est connue de la Sierra Nevada entre $900 \mathrm{~m}$ et $2400 \mathrm{~m}$ (Bertrand 1956, Serra-Tosio 1970).

Bertrand (1950 a, 1950 b) la signale des HautesPyrénées (Cauterets, Gavarnie) et des Pyrénées Atlantiques (Licq Atherey).

Nouvelles récoltes pyrénéennes:

- Rivière Touyre à $670 \mathrm{~m}$ (Brouquet-Laglaire 1985) ;

- Vallée d'Aure à 1000 m-1200 m (Gazagnes \& Laville 1983, sub Borcoheptagyia sp.);

- Vallée d'Ossau: Gave de Bious (1600 m).

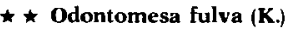

Ce Prodiamesinae d'un gen re monospécifique est connu d'Europe centrale - Hongrie, Tchécoslovaquie, Belgique, Allemagne, Sud-Suède (Brundin 1956), Roumanie (Cure 1985).

Nous l'avons récemment récolté dans deux affluents de la Somme, la Selle et l'Authie, des plaines du Nord de la France (Coll. Bazerque).

Récemment signalé dans la partie alpine du fleuve Rhin, en amont du Bodensee (Wilson \& Wilson 1985).

Il s'agit d'une espèce psammorhéophile (Cure loc. cit.) et plutôt tolérante à la pollution.

Dans les Pyrénées elle n'a été récoltée qu'en basse altitude : Vallée d'Aure à $420 \mathrm{~m}$ et $610 \mathrm{~m}$, Vallée d'Ossau dans le ruisseau lent d'Arriou Torı à $880 \mathrm{~m}$.

\section{Chaetocladius gracilis Br.}

Ce Chaetocladius signalé pour la première fois d'un émissaire du Lac Vassijaure dans la région de Tornetrask, en Laponie Suédoise (Brundin 1956) a été retrouvé dans les Pyrénées: Torrent d'Estaragne à $1920 \mathrm{~m}$ (Laville \& Lavandier 1977) et déversoir du lac d'Arrious à $2090 \mathrm{~m}$ dans la Vallée d'Ossau (Vinçon 1987).

I] présente actuellement une répartition boréoalpine.

\section{^ Cricotopus (Nostococladius) lygropis Edw.}

Repartition : Grande-Bretagne, Irlande, Suède (Ashe \& Murray 1980). Les larves de ce Cricotopus peu souvent cité, vivent à l'intérieur de colonies de l'algue bleu-verte Nostoc parmelioides Kutz.

En France, signalé de la Bourne, affluent de l'Isère (Serra-Tosio 1977) et de la rivière Lot à $443 \mathrm{~m}$ (Laville 1981). Nouvelle récolte dans les Pyrénées: Vallée d'Ossau, dans un ruisseau affluent du Gave de Bious à $1610 \mathrm{~m}$.

\section{Eukiefferiella cyanea Th.}

Espèce rhéobionte des massifs montagneux de moyenne altitude (Lehmann 1972).

Répartition : Laponie de Norvège $(1100 \mathrm{~m})$, Mittelgebirge de R.F.A., Alpes de Haute-Bavière $(1450 \mathrm{~m})$, Pyrénées (Lehmann loc. cit.), Carpates (Fittkau \& Reiss 1978).

Elle est signalée en Russie du Rhithral des petites montagnes du Caucase, de $950 \mathrm{~m}$ au niveau de la Mer Noire, près de la frontière turque (Kownacki \& Zosidge 1980).

Récentes récoltes du Bassin méditerranéen : Liban : rivière Oronte à $1400 \mathrm{~m}$ et $650 \mathrm{~m}$ (Moubayed \& Laville 1983); 
Tunisie du N-O: Oued Barbar-Kroumirie (Coll. Boumaiza).

Pyrénées :

- Gave de Pau à Gavarnie (1300 m) (Bertrand 1950);

- Rivière Touyre ( $450 \mathrm{~m}$ - $670 \mathrm{~m})$ (Brouquet-Laglaire 1985) :

- Vallée d'Aure (650 - $1440 \mathrm{~m}$ ) (Gazagnes \& Laville 1985, Bouguenec \& al 1984);

- Rio Garona à Salardu (1100 m) (Bertrand 1952).

- Vallée d'Ossau : Gave d'Ossau (490 m) et affluent du ruisseau de la Glère $(1980 \mathrm{~m})$.

\section{$\star \star$ Eukiefferiella pseudomontana G.}

Syn. E. ruthneri Gowin 1943.

Espèce assez peu souvent citée, connue seulement des Alpes françaises, autrichiennes et bavaroises (Gowin 1943).

Probablement crénophile à rhéophile, psychrosténotherme et polyoxybionte (Caspers 1983).

En France, signalée du Métarhithral de la rivière Lot dans le Massif Central (Laville 1981) et de la rivière Touyre $(600 \mathrm{~m})$ dans les Pyrénées (Brouquet-Laglaire 1985).

Récemment signalée dans trois rivières du Liban entre $650 \mathrm{~m}$ et $1200 \mathrm{~m}$ (Moubayed \& Laville 1983).

\section{$\star \star$ Heterotanytarsus apicalis $(\mathbf{K}$.)}

Répartition : Suede, Irlande, Grande-Bretagne, Allemagne du Nord, Tchécoslovaquie, Alpes de HauteBaviète (Brundin 1956).

Signalée de lacs oligotrophes de Suède, elle peut également devenir rhéophile dans les massifs montagneux comme les Carpates (Cure 1985) ou les Pyrénées - Vallée d'Ossau : torrent du Valentin $(1370 \mathrm{~m})$ en amont de Gourette.

\section{$\star \star$ Krenosmittia camptophleps (Edw.)}

Répartition : Laponie suédoise, Grande-Bretagne, Belgique, Alpes de Haute-Bavière et de BasseAutriche (Brudin 1956), Balkans (Caspers 1983), Carpates (Cure 1985).

Son écologie est peu connue : elle est certainement plus rhéobionte que $K$. boreoalpina - crénobionte et psychrosténotherme - avec qui elle paraît vicariante dans la Neste d'Aure (Gazagnes \& Laville 1985). La larve vit dans les mousses des sources et des rivières (Cure 1985).

Dans le Massif-Central elle a été signalée du Crénal au Métarhithral du Lot (Laville 1981) et de la source de l'Aveyron $(670 \mathrm{~m})$ (Viaud-Chauvet 1982).
Pyrénées :

- Rivière Touyre $(670 \mathrm{~m})$ (Brouquet-Laglaire 1985) ;

- Neste d'Aure (1080 m à $1400 \mathrm{~m}$ ) (Gazagnes \& Laville 1985) :

- Vallée d'Ossau : Torrent du Valentin $(1370 \mathrm{~m})$ et Gave de Brousset $(1490 \mathrm{~m})$.

\section{$\star$ Orthocladius (Euorthocladius) saxosus (Tok.)}

Répartition: Japon (Tokunaga 1939), Laponie suédoise (Thienemann 1944), URSS (Pankratova 1970), Balkans orientaux, dans plusieurs affluents du Danube de Roumanie, (Cure 1985) et Italie (Rossaro 1978).

Massif central : Rivière Lot $(900-1100 \mathrm{~m})$ (Laville 1981).

Les espèces du sous-genre Euorthacladius Th. sont rhéobiontes et colonisent les pierres des rivières à courant fort (Brundin 1956).

Pyrénées:

- Rivière Touyre $(670 \mathrm{~m})$;

- Vallée d'Ossau : Gave de Bious (1600 m).

\section{$\star \star$ Parametriocnemus boreoalpinus Gow.}

Cette espèce boréoalpine n'est connue que de Fenno-Scandinavie, des Alpes et des Carpates (Fittkau \& Reiss 1978) ; elle est signalée pour la première fois des Pyrénées, à basse altitude $(495 \mathrm{~m})$ dans le Canceigt, petit affluent du Gave d'Ossau.

\section{$\star \star$ Rheosmittia spinicornis (Br.)}

Syn. Parakiefferiella (Rheosmittia) spinicornis $\mathrm{Br}$. (Cranston \& Saether 1986).

Répartition : Suède : Fleuve Varke (Brundin 1956), Italie : Fleuve Pô (Rossaro 1977), Alpes de HauteBavière à $620 \mathrm{~m}$ (Reiss 1982), Carpates roumaines : vallée d'Olt et Monts Lotru (Cure 1985), Danemark (Cranston \& Saether loc. cit.), Highlands d'Ecosse (Langton 1984), Partie alpine du fleuve Rhin (Wilson \& Wilson 1985).

La plupart de ces récoltes dans des grands fleuves révèlent le caractère plutôt potamique de cette espèce.

Pyrénées: Vallée d'Ossau : Gave de Bious (1600 m).

\section{* Paracladopelma mikiana G.}

Syn. Paracladopelma schlitzensis Ringe 1974. Répartition : ce Chironomini apparait comme un élément méditerranéo-paléarctique dont la limite septentrionale se situe aux chaînes des Pyrénées, des Alpes et des Carpates. 
Signalée de l'épirhithral du Breitenbach, petite rivière de plaine d'Allemagne et $d^{\prime} u n e$ rivière des Basses-Alpes près de Forcalquier $(500 \mathrm{~m}$ ) (Ringe 1974).

Pyrénées : deux stations dans la Neste d'Aure à 1080 m et $1400 \mathrm{~m}$ (Gazagnes \& Laville 1985).

Citée également du Bassin méditérranéen : Grèce (Reiss 1977) et Turquie (Reiss 1985).

\section{Krenopsectra fallax Reiss}

Cette espèce, psychrosténotherme, vit dans les mousses des sources et des rivières de montagne. Elle n'est connue que des Alpes de Basse-Autriche (Lunz) et vraisemblablement de Suisse (Parc National d'Engadin) et, dans les Pyrénées, du Val d'Enfer (Reiss 1969) et de Barèges, sous la cascade d'un ruisselet (Bertrand 1950b, sub Gowiniella sp.).

Nouvelle station dans la Vallée d'Ossau : Gave de Bious $(1600 \mathrm{~m})$.

\section{^ Rheotanytarsus pentapoda $K$.}

Ce Tanytarsini est signalé de plusieurs rivières des Mittelgebirge (Hasperbach, Daun, Fulda), de ForêtNoire et du Lac Majeur (Lehmann 1970).

Répartition : Allemagne, Italie (Lehmann loc. cit.), URSS (Pankratova 1983). Irlande (Murray \& Ashe 1980), Grande Bretagne (Langton 1984).

Ses exigences écologiques sont peu connues : les larves construisent des fourreaux caractéristiques sur les pierres et les Ranunculus en courant lent ou rapide (Ashe 1982).

Citations en France : S-O du Massif-Central dans le Crénal et l'Hyporhithral du Lot (Laville 1981), dans l'Epipotamal de l'Aveyron à Laissac $(570 \mathrm{~m})$ (Viaud. Chauvet 1982) et dans deux affluents pollués du Tarn, l'Agout (aval Graulhet) et le Dadou (aval Castres) (Brouquet-Laglaire 1985).

Pyrénées :

- Rivière Touyre (670 m) (Brouquet-Laglaire loc. cit.) ; - Neste d'Aure à $610 \mathrm{~m}$;

- Vallée d'Ossau: Gave d'Ossau à $510 \mathrm{~m}$.

\section{Conclusion}

La connaissance actuelle de la faune chironomidien:ne des Pyrénées permet de dénombrer 288 espèces réparties dans 100 genres différents. On ne peut l'estimer par rapport à la Faune de France dont il n'existe aucun inventaire actualisé.
Elle représente $24 \%$ de la Faune européenne qui comprend quelques 1200 espèces pour 186 genres.

La faune pyrénéenne peut également être compa rée avec celles récemment inventoriées de GrandeBretagne ( 460 espèces), d'Irlande (347 espèces) et de Roumanie ( 407 espèces) dont elle se rapproche respectivement à $63 \%, 83 \%$ et $70 \%$.

La prospection intensive de milieux stagnants de basse et moyenne altitude ainsi que celle d'habitats plus chauds de la partie orientale de la chaine devraient encore permettre d'élargir cet inventaire faunistique des Chironomidés des Pyrénées.

\section{Travaux cités}

Ashe (P.). 1982. - Ecological and taxonomic studies on the Chironomidae (Diptera. Nematocera). I : A study of the Chironomidae of the river Flesk, S.W. Ireland. Ph. D. Thesis. Univ. Dublin : $158 \mathrm{p}$.

Ashe (P.) \& Murray (D.A.). 1984. - An inventory of the Irish Chironomidae (Diptera), Mem. Amer. Ent. Soc., 34: 223-233.

Bertrand (H.). 1950a. - Chirnomides pyrénéens nouveaux pour la faune française. Bull. Soc. ent. Fr., $55: 11-12$.

Bertrand (H.). 1950b. - Diptères Chironomides pyrénéens. Buil. Soc. ent. Fr. $55: 153-155$.

Bertrand (H.). 1952. - Récoltes de Diptères Chironomides dans les Pyrénées. (2“ note). Vie et Milieu, 3 (3): 314-321.

Bertrand (H.). 1956. - Diptères Chironomides pyrénéens et espàgnols. Bull. Soc. ent. Fr., $61 ; 93-95$.

Bouguenec (V.), Meurgues (S.) \& Sourzac (C.). 1984. Introduction à l'étude d'une rivière pyrénéeme aménagée : la Neste d'Aure. D.E.A. Ecologie, Toulouse III : $159 \mathrm{p}$.

Brouquet-Laglaire (Y.). 1985. - Etude des Chironomidés (Diptera) de quelques rivières polluées du bassin de la Garonne: Touyre, Agout, Dadou, Thoré, Bas-Lot. Thèse $3^{e}$ cycle, Ecologie, Tou. louse III : $237 \mathrm{p}$.

Brundin (L.). 1956. - Zur Systematik der Orthocladiinae (Dipt Chironomidae). Rep. Inst. Freshwat. Res. Drotmingholm, 37 5.185.

Caspers (N.). 1983. - Chironomiden-Emergenz zweier Lunzer Bäche, 1972, Arch. Hydrobiol./Suppl. 65 (4) : $484-549$.

Cranston (P.S.) \& Saether (O.A.). 1986. - Rheosmittia (Diptera Chironomidae) : a generic validation and revision of the western Palaearctic species, Journal of Natural History, $20: 31-51$.

Cure (V.). 1985. - Chironomidae (Diptera-Nematocera) aus Rumänien unter besonderer Beriicksichtigung jener aus dem hydrographischen Einzugsgebiet der Donau. Arch. Hydrobiol.Suppl., $68: 163-217$,

Fittkau (E.J.) \& Murray (D.A.). 1983. - Pentaneurella katteriokki, eine neue Gattung und Art der Tanypodinae. Nachrichtenblatt der Baverischen Entomologen, 32 (2) : 57-63.

Fittkau (E.J.) \& Reiss (F.). 1978. - Chironomidae. in Illies J. ed. Limnofauna Europaea: $404-440$, G. Fisher, Stuttgart.

Gazagnes (G.) \& Laville (H.). 1985. - Etude faunistique des Chironomidés (Diptera) de la Haute Neste d'Aure (Pyrénées Centrales): impact des aménagements hydroélectriques, Annls Limnol. 21 (2) : 149-159.

Gonzales Peña (G.). 1983. - Factores que determinan la distribu. ción de los simúlidos y quironomidos (Diptera) de la red hidro gráfica andorrana. Actas del primer congress espanol de Limnologia: 197-205. 
Gowin (F.). 1943. - Orthocladiinen aus Lunzer Fliessgewässern II. Arch. Hydrobiol., $40: 114.122$.

Kownacki (A.) \& Zosidge (R.S.). 1980. - Taxocens of Chironomidae (Diptera) in some rivers and streams of the Adzhar ASSR (Little Caucasus Mts). Acta Hydrobiol. 22 (1): 67-87.

Langton (P.H.). 1984. - A key to pupal exuviae of British Chironomidae, P.H. Langton ed. Cambridgeshire : $323 \mathrm{p}$

Laville (H.) 1966. - Chironomides du Massif de Néouvielle (Pyró. nées centrales) (Diptères). Annis Limnol. 2(1): 203-216.

Laville (H.) 1978. - Inventaire des Chironomides connus des Pyrénèes en 1976. Acta Univ. Carol.Biol. 1980, 12:103-113.

Laville (H.). 1979. - Etude de la dérive des exuvies nymphales de Chironomides au niveau du confluent Lot-Truyere. Annls Limnol. 15 (2) : 155-180.

Laville (H.). 1980. - Inventaire 1980 des Chironomides (Diptera) connus des Pyrénées. Amnls Limnol., 16 (3) : 211-223.

Laville (H.) 1981. - Récoltes d'exuvies nymphales de Chironomides (Diptera) dans le Haut-Lot, de la source (1295 m) au confluent de la Truyere (223 mi). Annls. Limnol, 17 (3) : 225-289.

Laville (H.) \& Lavandier (P.). 1977. - Les Chironornides (Diptera) d'un torrent pyrénéen de haute montagne: l'Estaragne. Annls Limnol, 13 (1): 57-81.

Lehmann (I.). 1970. - Revision der europäischen Arten (Imagines $\sigma$ und Puppen o) der Gattung Rheotanytarsus Bause (Diptera, Chironomidae) Zool. Anz., 185:345-378

I.ehmann (J.). 1971. - Die Chironomiden der Fulda (Systematische, ökologische und faunistische Untersuchungen). Arch. Hydrobiol.Suppl., $37: 466-555$.

Lehmann (J.). 1972. - Revision der europaïschen Arten (Puppen und Imagines) der Gattung Eukiefferiella Thienemann (Diptera, Chironomidae). Beitr. Ent., 22: 347-405.

Moubayed (Z.) \& Laville (H.). 1985. - Les Chironomidés (Diptera) du Liban. I. Premier inventaire faunistique, Anmls Limnol., 19 (3) : $219-228$.

Murray (DA.) \& Ashe (P.). 1980. - Nostococladius, a new subgenus of Cricotopus (Diptera : Chironomidae). In Chironomidae: Ecology, Systemaics, Cytology and Physiology. Murray d.a.ed., Pergamon Press, Oxford and New-York: 105.111.

Pankratova (V. Ya). 1970. - Larvae and pupae of midges of the subfamily Orthocladiinae (Diptera, Chironomidae : Tendipedi. dae) of the USSR fauna (en Russe), $1 z d$. Nauka, Leningr., $344 \mathrm{p}$.

Pankratova (V. Ya). 1983. - Lariae and pupae of midges of the subfamily Chironomidae (Diptera, Chironomidae : Tendipedi. dae) of the USSR fauna (en Russe), Izd. Nauka, Leningr., $296 \mathrm{p}$.

Prat (N.). 1981. - Quironomidos de Cataluñya. (2 a Nota), Mediterranea, $5: 43-66$

Prat (N.), Puig (M.A.). Gonzalez (G.) \& Millet (X.). 1984. Chironomid longitudinal distribution and macroinvertebrate diversity along the Llobregat River (NE Spain), Mem. Amer. Ent. Soc., 34:267-278.
Reiss (F.). 1969. - Krenopsectra fallax gen. n. sp. n. (Diptera, Chironomidae) aus den Alpen und Pyrenäen, Ann. Zool. Fennici, $6: 435-442$.

Reiss (F.) 1977. - Verbreitungsmuster bei paläarktischen Chironomidenarten (Diptera, Chironomidae), Spixiana, 1 (1) : 85.97

Reiss (F.). 1982. - Chironomidenfauna des Murnauer Mooses in Oberbayem (Insccta, Diptera), EntomofaundSupp., 1 : 263-288.

Reiss (F.) 1985. - A contribution to the zoogeography of the tur kish Chironomidae (Diptera), Israel Jounial of Ettomology, XIX : 161-170.

Rieradevall I Sant (M.). 1985. - Ritme diari de la deriva en una estacio del riu Llobregat, amb especial attentio a les exuvies pupals dels Chironomidae (Dipt.). Tesi de Llicenciatura, Uni versitat de Barcelona : $175 \mathrm{p}$.

Ringe (F.). 1974. - Chironomiden-Emergenz 1970 in Breitenbach und Rohrwiesenbach. Schlitzer Produktions-biologische Studien 10. Arch. Hydrobiol.Suppl., 45: 212-304.

Serra-Tusio (B.). 1970. - Some Diamesinae from Southern Spain (Insecta, Diptera, Chironomidae). Steemstrupia, 1 : 25-27.

Thienemann (A). 1944. - Bestimmungstabelle für die bis jetzt bekannten Larven und Puppen der Orthocladiinae (Diptera, Chironomidae). Arch. Hydrobiol, $39: 551.664$

Rossaro (B.). 1977. - Note sulle Orthocladiinae italiane con segnalazione di specie nuove per la nostra fauna. Boll. Soc. entom. ital., $109(7.8): 117-127$.

Rossaro (B.). 1978. - Contributo alla conoscenza dei generi Ortho cladius, Parorthocladius e Synorthocladius rassegna delle specie catturate sinora in Italia. Boll. Soc. entom. ital., 110 (9) : $181-188$.

Thomas (A.G.B.). 1981. - Travaux sur la taxonomie, la biologie et l'écologie d'insectes torrenticoles du Sud-Ouest de la France (Ephéméroptères et Diptères : Dixidae, Cecidomydae, Rhagionidac et Athericidae) avec quelques exemples de perturbations par l'Homme. These Doct. Etat. Toulouse III : $320 \mathrm{p}$.

Tokunaga (M.). 1939. - Chironomiden from Japan (Diptera) XI. New or little-known midges, with several descriptions of the metamorphoses of torrential species. Philipp. J. Sci, 69: 297-345.

Viaud-Chauvet (M.). 1982. - Analyse des récoltes d'exuvies nymphales de Chironomides dans les rivières du bassin du Tarn. Thèse de 3e cycle, Ecologie, Toulouse IHI : $171 \mathrm{p}$.

Vinçon ( $G$.). 1987. - Comparaison de la faune benthique des vallées d'Aure et d'Ossau, en vue de l'élaboration d'une méthodo. logie de surveillance des cours d'eau de montagne. Thèse de Docteur-Ingénieur, Ecologie, Toulouse III : 381 p.

Wilson (R.S.) \& Wilson (S.E.). 1985. - A reconnaissance of the river Rhine using Chironomidae pupal exuviae (Insecta : Diptera). Mem. Amer. Ent. Sac, $34: 361.385$. 